Si ngl e- I ayer ed Compl ex- Val ued Neur al Net wor $k$ for Real - Val ued $\mathrm{Cl}$ assi fi cat i on Probl ens

\begin{tabular}{|l|l|}
\hline 著者 & AM N Fai $\mathrm{j}$ ul , MURASE Kazuy uki \\
\hline $\begin{array}{l}\text { j our nal or } \\
\text { publ i cat i on t i t l e }\end{array}$ & Neur ocomput i ng \\
\hline vol une & 72 \\
\hline number & $4-6$ \\
\hline page r ange & $945-955$ \\
\hline year & $2009-01$ \\
\hline URL & ht t p: //hdl . handl e. net /10098/2090 \\
\hline
\end{tabular}




\title{
Single-layered Complex-Valued Neural Network for Real-Valued Classification Problems
}

\author{
Faijul Amin ${ }^{1}$ and Kazuyuki Murase $e^{1,2}$ \\ ${ }^{1}$ Department of Human and Artificial Intelligence Systems, Graduate School of Engineering, \\ and ${ }^{2}$ Research and Education Program for Life Science, University of Fukui, 3-9-1 Bunkyo, \\ Fukui 910-8507, Japan
}

Acknowledgement: Supported by grants of K.M. from Japanese Society for promotion of Sciences, and Technology, and the University of Fukui.

\author{
Correspondence: Dr. Kazuyuki Murase \\ Professor \\ Department of Human and Artificial Intelligence Systems, University \\ of Fukui \\ 3-9-1 Bunkyo, Fukui 910-8507, Japan \\ Tel: $+81(0) 776278774$ \\ Fax: +81(0)776278420 \\ E-mail: murase@synaspe.his.fukui-u.ac.jp
}

The total number of pages 35, figures 6 , tables 9 .

The total number of words in the whole manuscript 5281, abstract 242, introduction 605 


\title{
Single-layered Complex-Valued Neural Network for Real-Valued Classification Problems
}

\begin{abstract}
This paper presents two models of complex-valued neurons (CVNs) for real-valued classification problems, incorporating two newly-proposed activation functions, and presents their abilities as well as differences between them on benchmark problems. In both models, each real-valued input is encoded into a phase between 0 and $\pi$ of a complex number of unity magnitude, and multiplied by a complex-valued weight. The weighted sum of inputs is fed to an activation function. Activation functions of both models map complex values into real values, and their role is to divide the net-input (weighted sum) space into multiple regions representing the classes of input patterns. The gradient-based learning rule is derived for each of the activation functions. Ability of such CVNs are discussed and tested with two-class problems, such as two and three input Boolean problems, and symmetry detection in binary sequences. We exhibit here that both the models can form proper boundaries for these linear and nonlinear problems. For solving $n$-class problems, a complex-valued neural network (CVNN) consisting of $n$ CVNs is also considered in this paper. We tested such single-layered CVNNs on several real world benchmark problems. The results show that the classification and generalization abilities of single-layered CVNNs are comparable to the conventional real-valued neural networks (RVNNs) having one hidden layer. Moreover, convergence of CVNNs is much faster than that of RVNNs in most of the cases.
\end{abstract}

Keywords: activation function, classification, complex-valued neural networks, generalization, phase-encoding. 


\section{Introduction}

Complex numbers are used to express real-world phenomena like signal's amplitude and phase, and to analyze various mathematical and geometrical relationships. In order to directly process complex values by artificial neural networks, the complex-valued neural network $(\mathrm{CVNN})$ as well as the complex back-propagation (CBP) algorithm have been developed [3, $7,8,10,16]$. Properties of CVNN and CBP have been studied $[5,11]$, and CVNN is shown to be powerful in applications such as adaptive radar image processing [15], and optical image processing $[4,2]$. Further extension to multi-dimensional values has been attempted as well [13]. Some researchers recently have applied CVNN on real-valued classification problems too $[1,9]$.

There have been two approaches for the application of CVNN on real-valued classification problems as of our knowledge. In [9], each real-valued input is phase encoded between 0 and $\pi / 2$ of unity magnitude complex number, so that the complex-valued neuron (CVN) can receive complex-valued inputs. The role of $\mathrm{CVN}$ is two-fold, aggregation and threshold operations. The former role is to aggregate the inputs multiplied by connection weights, and the latter is to determine the class label by using activation function. They showed that the $\mathrm{CVN}$ is successful to classify all two-input Boolean functions, and 245 among 256 threeinput Boolean functions. The learning algorithm, however, includes reciprocal of partial derivatives. When the partial derivatives go near zero and the reciprocals become very large, the learning process may become unstable, especially when real world problems are dealt. In a recent work [1], a multilayer feed-forward architecture of multi-valued neuron is proposed. The model encodes real-valued inputs by phases between 0 and $2 \pi$ of unity magnitude complex numbers, and determines the class label by complex-valued output, based on 
output's vicinity to the roots of unity. It has been shown that the model was able to solve the parity $n$ and two spirals problems, and could perform better in "sonar" benchmark and the Mackey-Glass time series prediction problems.

In this paper, we propose two activation functions that map complex values to real-valued outputs. The role of the activation functions is to divide the net-input (sum of weighted inputs) space into multiple regions representing the classes. Both the proposed activation functions are differentiable with respect to real and imaginary parts of the net-input. As a result, gradient-based learning rule can be derived. To present complex-valued inputs to CVN, real-valued inputs are phase encoded between 0 and $\pi$. We will show in the following sections that such a CVN is able to solve linear and nonlinear classification problems such as two-input Boolean functions, 253 among 256 three-input Boolean functions, and symmetry detection in binary sequences.

To solve $n$-class problems, we considered a single-layered CVNN (without hidden layer) consisting of $n$ CVNs described above, and formulated the learning and classification scheme. The single-layered CVNN has been applied and tested on various real world benchmark problems. Experimental results showed that generalization ability of the single-layered CVNN is comparable to the conventional two-layered (with one hidden layer in addition to input and output layer) real-valued neural network (RVNN). It is noteworthy that in the proposed single-layered CVNN, the architecture selection problem does not exist. In the multilayered RVNNs which are considered as universal approximators [6], in contrast, the architecture selection is crucial to achieve better generalization and faster learning abilities. 
The remaining of the paper is organized as follows. In section 2, we discuss about the model of $\mathrm{CVN}$ along with the proposed activation functions. In section 3, we develop gradientbased learning rules for training the CVNs. Section 4 discusses the ability of the single CVN on some binary-valued classification problems. In section 5, performances of the singlelayered CVNN consisting of multiple CVNs are compared to those of the conventional twolayered RVNN on some real world benchmark problems. Finally, section 6 gives the discussion and concluding remarks.

\section{Complex-valued neuron (CVN) model}

Since the complex-valued neuron $(\mathrm{CVN})$ processes complex-valued information, it is necessary to map real input values to complex values for solving real-valued classification problems. After such mapping, the neuron processes information in a way similar to the conventional neuron model except that all the parameters and variables are complex-valued, and computations are performed according to the complex algebra. As illustrated in Fig. 1, the neuron, therefore, first sums up the weighted complex-valued inputs and the threshold value to represent its internal state for the given input pattern, and then the weighted sum is fed to an activation function which maps the internal state (complex-valued weighted sum) to a real value. Here, the activation function combines the real and imaginary parts of the weighted sum.

\subsection{Phase encoding of the inputs}

This section explains how the real-valued information is presented to a CVN. Consider $(X, c)$ be an input example, where $X \in R^{m}$ represents the vector for $m$ attributes of the example, and $c \in\{0,1\}$ denotes the class of the input pattern. We need a mapping $R^{m} \rightarrow C^{m}$ to 
process the information by $\mathrm{CVN}$. One such mapping for each element of the vector $X$ can be done by the following transformation.

$$
\begin{aligned}
& \text { Let } x \in[a, b] \text {, where } a, b \in R, \text { then } \\
& \varphi=\pi(x-a) /(b-a), \text { and } \\
& z=e^{i \varphi}=\cos \varphi+i \sin \varphi
\end{aligned}
$$

Here $i=\sqrt{-1}$. Eq. (1) is a linear transformation which maps $x \in[a, b]$ to $\varphi \in[0, \pi]$. Then by the Euler's formula, as given by Eq. (2), a complex value $z$ is obtained. When a real variable moves in the interval of $[a, b]$, the above transformation will move the complex variable $z$ over the upper half of a unit circle. As shown in Fig. 2, the variation on a real line is thus now in the variation of phase $\varphi$ over the unit circle.

Some facts about the transformation are mentionable. Firstly, the transformation retains relational property. For example, when two real values $x_{1}$ and $x_{2}$ hold a relation $x_{1} \leq x_{2}$, the corresponding complex values have the same property in their phases as such, $\operatorname{phase}\left(z_{1}\right) \leq \operatorname{phase}\left(z_{2}\right)$. Secondly, the spatial relationship between real values is also retained. For example, two real values $x_{1}$ and $x_{2}$ are farthest from each other when $x_{1}=a$ and $x_{2}=b$. The transformed complex values $z_{1}$ and $z_{2}$ are also farthest from each other as shown in Fig. 2 . Thirdly, the interval $[0, \pi]$ is better than the interval $[0,2 \pi]$ as we loose the spatial relationship among the variables in the latter. For example, $x_{1}=a$ and $x_{2}=b$ will be mapped to same complex value, since $e^{i 0}=e^{i 2 \pi}$. It is mentionable that interval $[0, \pi / 2]$ can also be used for phase encoding. However, experiments presented in the section 5 and Table 9 exhibit that learning convergence is faster in case of the interval [0, 
$\pi]$. Finally, the transformation can be regarded as a preprocessing step. The preprocessing is commonly used even in real-valued neural networks in order for mapping input values into a specified range, and so on. The transformation in the proposed $\mathrm{CVN}$, therefore, does not increase any additional stage for the process with neural networks. In fact, the above transformation does not loose any information from the real values, rather lets a CVN to process the information in a more powerful way.

\subsection{Activation function}

When a CVN sums up inputs multiplied by the synaptic weights, the weighted sum is fed to an activation function to make the output bounded. In the real-valued case, activation functions are generally differentiable and bounded in the entire real domain, which makes it possible to derive gradient-based backpropagation (BP) algorithm. But from Liouville's theorem, there is no complex-valued function except constant which is differentiable and bounded at the same time. However, this is not as frustrating as it seems. For solving realvalued classification problems, we can combine real and imaginary parts of the weighted sum in some useful ways.

In this paper we propose two activation functions that map complex values to real values for classification problems. Their role is to divide the neuron's net-input (sum of weighted inputs) space into multiple regions for identifying the classes, as is the role of the real-valued neuron in classification problems.

Let the net-input of a complex neuron be $z=u+i v$, then we define two activation functions 1 and 2 by the following Eq. (3) and Eq. (4), respectively. 


$$
\begin{aligned}
& f_{C \rightarrow R}(z)=\sqrt{\left(f_{R}(u)\right)^{2}+\left(f_{R}(v)\right)^{2}} \\
& f_{C \rightarrow R}(z)=\left(f_{R}(u)-f_{R}(v)\right)^{2}
\end{aligned}
$$

where $f_{R}(x)=1 /(1+\exp (-x))$ and $x, u, v \in R$. Both the activation functions combine the real and imaginary parts, but in different ways. The real and imaginary parts are first passed through the same sigmoid function individually. Thus each part becomes bounded within the interval $(0,1)$. The activation function 1 in Eq. (3) gives the magnitude of the resulted complex value, while the activation function 2 in Eq. (4) gives the square of the difference between real and imaginary parts. Fig. 3 illustrates the output value $y$ of both the activation functions in the domain of real and imaginary parts of net-input, $u$ and $v$, respectively. It is noteworthy that defining activation functions as above, makes it possible to compute the partial

derivatives $\frac{\partial f}{\partial u}$ and $\frac{\partial f}{\partial v}$, which in turn makes it possible to derive gradient-based learning rules.

As seen in Fig. 3, both the CVNs with the proposed activation functions saturate in four regions. On the contrary, the real-valued sigmoid function saturates only in two regions. Thus a real valued neuron can solve only linearly separable problem. We show in the later sections that saturation in four regions of the proposed CVNs significantly improves their classification ability.

\section{Learning rule}

Here we derive a gradient-descent learning rule for a CVNN consisted of multiple CVNs described in the previous section. We deal with the CVNN without any hidden units. 
Consider an $m-n$ CVNN, where $m$ and $n$ are the numbers of inputs and outputs, respectively. Let $X=\left[x_{1} \cdots x_{m}\right]^{T}$ be an $m$ dimensional complex-valued input vector, $W_{k}=\left[w_{k 1} \cdots w_{k m}\right]^{T}$ the complex-valued weight vector of neuron $k$, and $\theta_{k}$ the complex-valued bias for the $k$ th neuron. To express the real and imaginary parts, let $x_{j}=x_{j}^{R}+i x_{j}^{I}, w_{k j}=w_{k j}^{R}+i w_{k j}^{I}, \theta_{k}=\theta_{k}^{R}+i \theta_{k}^{I}$. Then the net-input $z_{k}$, and the output $y_{k}$ of the $k$ th neuron are given by

$$
\begin{aligned}
z_{k} & =\sum_{j=1}^{m} w_{k j} x_{j}+\theta_{k} \\
& =\left(\sum_{j=1}^{m}\left(w_{k j}^{R} x_{j}^{R}-w_{k j}^{I} x_{j}^{I}\right)+\theta_{k}^{R}\right)+i\left(\sum_{j=1}^{m}\left(w_{k j}^{I} x_{j}^{R}+w_{k j}^{R} x_{j}^{I}\right)+\theta_{k}^{I}\right) \\
& =z_{k}^{R}+i z_{k}^{I}
\end{aligned}
$$

and

$$
y_{k}=f_{C \rightarrow R}\left(z_{k}\right)
$$

If the desired output of the $k$ th neuron is $d_{k}$, then the error function to be minimized during the training is given by

$$
E=(1 / 2) \sum_{k=1}^{n}\left(d_{k}-y_{k}\right)^{2}=(1 / 2) \sum_{k=1}^{n} e_{k}^{2}
$$

During the training, the biases and the weights are updated according to the following equations.

$$
\begin{aligned}
& \Delta \theta_{k}=-\eta \frac{\partial E}{\partial \theta_{k}^{R}}-i \eta \frac{\partial E}{\partial \theta_{k}^{I}}=\Delta \theta_{k}^{R}+i \Delta \theta_{k}^{I} \\
& \Delta w_{k j}=-\eta \frac{\partial E}{\partial w_{k j}^{R}}-i \eta \frac{\partial E}{\partial w_{k j}^{I}}=\Delta w_{k j}^{R}+i \Delta w_{k j}^{I}=\bar{x}_{j} \Delta \theta_{k}
\end{aligned}
$$

where $\eta$ is the learning rate and $\bar{x}_{j}$ is the complex conjugate of the complex number $x_{j}$.

If the activation function is given by Eq. (3), then 


$$
\begin{aligned}
& \Delta \theta_{k}^{R}=\eta e_{k} \frac{f_{R}\left(z_{k}^{R}\right)}{y_{k}} f_{R}^{\prime}\left(z_{k}^{R}\right) \\
& \Delta \theta_{k}^{I}=\eta e_{k} \frac{f_{R}\left(z_{k}^{I}\right)}{y_{k}} f_{R}^{\prime}\left(z_{k}^{I}\right)
\end{aligned}
$$

where $f_{R}(u)=1 /(1+\exp (-u))$ and $f_{R}^{\prime}(u)=f_{R}(u)\left(1-f_{R}(u)\right), u \in R$.

If the activation function is given by Eq. (4), then

$$
\begin{aligned}
& \Delta \theta_{k}^{R}=2 \eta e_{k}\left(f_{R}\left(z_{k}^{R}\right)-f_{R}\left(z_{k}^{I}\right)\right) f_{R}^{\prime}\left(z_{k}^{R}\right) \\
& \Delta \theta_{k}^{I}=2 \eta e_{k}\left(f_{R}\left(z_{k}^{I}\right)-f_{R}\left(z_{k}^{R}\right)\right) f_{R}^{\prime}\left(z_{k}^{I}\right)
\end{aligned}
$$

\section{Ability of CVN}

A CVN is more powerful than a real-valued neuron (RVN), since it is capable to solve linearly non-separable problems too. This ability is explained in this section for two and three input Boolean functions, and for the symmetry detection in binary sequences.

\subsection{Two-input Boolean Problems}

There are 16 possible Boolean functions of two inputs. Some are linearly separable, while others are not. A RVN can solve only linearly separable problems since it gives a straight line as a decision boundary. To say this equivalently, activation function of such a neuron saturates only in two regions. But a CVN with the proposed activation functions saturates in four regions and thus can achieve higher classification ability. In fact, a CVN can solve all the two-input Boolean functions.

First, we explain it graphically for the XOR problem. Inputs $x_{1}$ and $x_{2}$ in Table 1 show the input values after phase-encoding discussed in section 2.1. Let the weighted sum or the net- 
input of the neuron be $z=w_{1} x_{1}+w_{2} x_{2}+\theta=u+i v$, where $\theta$ is the bias. After training the neuron, the net-input for each input pattern should produce the output near the desired output value shown in Table 1 by the mapping of $y=f_{C \rightarrow R}(z)$.

The net-inputs for the four input patterns in XOR problem and the contour diagram of output $y$ are shown in Fig. 4. Among the four regions of saturation in the activation function 1 (Fig. 4(a)), the net-inputs after training were located at three regions; upper left, lower left, and lower right regions, since the desired outputs were either zero or one. In case of the activation function 2 (Fig. 4(b)), all four regions were used. As apparent in Fig. 4, both CVNs with the proposed activation functions were able to solve the XOR problem, while a single RVN cannot solve such a linearly non-separable problem.

Parameters obtained after training, for all possible two-input Boolean functions, are given in Table 2. Each sequence $Y=y_{1} y_{2} y_{3} y_{4}$ denotes a Boolean function, where $y_{1}=f(0,0), y_{2}=f(0,1), y_{3}=f(1,0)$ and $y_{4}=f(1,1)$. For example, the sequence 0110 is the XOR problem, 0001 is the AND problem, 0111 is the OR problem, and so on.

\subsection{Three-input Boolean Problems}

For three inputs, the number of different Boolean functions is 256. Michel et al. [9] have reported that their CVN model solves 245 among the 256 functions. Our proposed model of CVN with the activation function 2 could solve 253 functions, and 250 functions with the activation function 1 . These impressive results are suggestive to examine the CVN, with the new activation functions, on more complex problems. 


\subsection{Symmetry detection Problem}

The symmetry detection problem is to detect whether or not binary activity levels of a onedimensional input array is symmetrical about the center point. An example of input-output mapping, for the input length of three bits is shown in Table 3. It is a linearly non-separable problem and hence can not be solved by a single RVN.

\subsubsection{Solving with one CVN}

We tested CVN on symmetry detection problems of various input lengths ranging from 2 bits to 10 bits. A single CVN with the proposed activation functions could solve all of the problems. For training the CVN, we set the target values as follows. With the activation function 1 , the target was set to 0 if the input pattern was symmetric, and 1 if asymmetric. With the activation function 2, target was 1 if symmetric, and 0 if asymmetric.

Solutions for four-input problem obtained with the activation function 1 and 2 are shown in the contour graphs (a) and (b) in Fig. 5, respectively. Though the activation function 1 saturates in four regions, we can expect that three regions will be used because the target output values are set to 0 and 1 . The other region for which output is near $\sqrt{2}$ will not be used. As expected, experimental results presented in Fig. 5(a) showed that the net-inputs for sixteen different input patterns were distributed in the three regions, according to symmetry and asymmetry, after the training. For the activation function 2, on the other hand, all four regions of saturation can be used, and the experimental results in Fig. 5(b) shows that the input patterns were distributed in four regions according to symmetry and asymmetry.

We then reversed the target output setting for both the activation functions. That is, with the activation function 1 , the target was set to 1 if the input pattern was symmetric, and 0 if asymmetric, while with the activation function 2 , target was set to 0 if symmetric, and 1 if 
asymmetric. The CVN with the activation function 1 could solve the symmetry detection problem up to the length of 3 bits, and with the activation function 2 up to 5 bits. This degraded result can be explained by the imbalance in numbers of input patterns assigned to each output value. That is, as the length of inputs increases, the percentage of symmetric inputs among all possible input patterns decreases. For example, there are $50 \%$ symmetric patterns for the symmetry detection problem of 3 bits, while only $6.25 \%$ and $3.125 \%$ for that of 9 and 10 bits, respectively. The detailed explanation is given below.

Nonlinearity comes from the fact that input patterns having numerically far different values, and being spatially far different in location belong to the same class. When the same weight vector is multiplied with different input vectors of the same class, the weighted sum is different. The activation function 1 has two regions of saturation for the target value of 1 and only one region for the target value of 0 (Fig. 3(a)). Since most of the input patterns are asymmetric for higher dimensional problems having the target output 0 , in case of reverse target setting, only one region is not enough to accommodate all asymmetric input patterns having numerically far different values. With the other target setting, 0 for symmetry and 1 for asymmetry, activation function 1 could solve higher dimensional problems as there are two well spread regions for output values near the value of 1 , and most of the input patterns are asymmetric having the target value of 1 . The same argument can be applied to the CVN with the activation function 2 . That is, in case of reverse target output settings ( 0 for symmetric and 1 for asymmetric), though there are two regions of saturation for target output of 1 , the regions are not as spread as the regions for 0 , while most of the input patterns are asymmetric. 


\subsubsection{Solving with two CVNs}

To solve the output encoding problem described above, one solution is to use two CVNs, each with opposite target output settings. If the target value for one CVN is 1 , then that of the other CVN is 0 . Output class of a given input pattern is decided according to the CVN with the larger output. Results of using two CVNs for the symmetry detection problems are summarized in Table 4. Here the average of 10 independent runs is shown. Two CVNs with activation function 1 could solve the symmetry detection problem up to seven bits, and activation function 2 up to six bits. The reason for these degraded results in comparison to using a single $\mathrm{CVN}$ is that although response from one of the $\mathrm{CVN}$ is near its target value, the output of the other neuron degrades the result more strongly. This can be seen from table 5, where a result of a single run for the seven-bit symmetry detection problem with the activation function 2 is given. Underlined outputs denote the misclassification. Response of one of the neuron (neuron 2) degraded the result strongly even though the other neuron (neuron 1) gave the correct output.

Nevertheless, though limitations exist, a single CVN is at least more powerful than a single $\mathrm{RVN}$ as it can solve linear as well as nonlinear problems to some considerable extents. Its applicability will be justified in the next section where we applied multiple CVNs on real world benchmark problems.

\section{Single-layered complex-valued neural network (CVNN) on real world classification problems}

Discussion in the previous sections is suggestive to explore the ability of multiple CVNs, which we call the single-layered complex-valued neural network (CVNN), on real world 
classification problems. To compare CVNN's performance with the conventional two-layered RVNN, both the neural networks are tested on some real world benchmark problems. We used Proben1 [14] datasets for the experiments. In this section, first we give a short description of the datasets, and then compare learning convergence and generalization ability of CVNN and RVNN.

\subsection{Description of the datasets}

PROBEN1 is a collection of learning problems well suited for supervised learning. Most of the problems are classification problems regarding diagnosis. Here we use five of them. Each of the problems is available in three datasets, which vary in the ordering of examples. For example, cancer problem consists of cancerl, cancer2 and cancer3 datasets. Short descriptions of these five problems are given below.

\subsubsection{Cancer}

This is a problem on diagnosis of breast cancer. The task is to classify a tumor as either benign or malignant based on cell descriptions gathered by microscopic examination. Input attributes are for instance the clump thickness, the uniformity of cell size and cell shape, the amount of marginal adhesion, and the frequency of bare nuclei. The data comprise of 9 inputs, 2 outputs, 699 examples, of which $65.5 \%$ are benign example. This data is originally obtained from University of Wisconsin Hospitals, Madison, from Dr. William H. Wolberg [17].

\subsubsection{Card}

The task is to predict the approval or non-approval of a credit card to a customer. Each example represents a card application and the output describes whether the bank (or similar institution) granted the card or not. There are 51 inputs, 2 outputs, and 690 examples.

\subsubsection{Diabetes}

This is on diagnosis of diabetes of Pima Indians. The task is to decide whether a Pima Indian individual is diabetes positive or not, based on personal data (age, number of times pregnant) 
and the results of medical examination (e.g. blood pressure body mass index, result of glucose tolerance test, etc.). It has 8 inputs, 2 outputs, and 768 examples.

\subsubsection{Glass}

Here the task is to classify glass types. The results of a chemical analysis of glass splinters (percent content of 8 different elements) plus the refractive index are used to classify the samples to be either float processed or non float processed building windows, vehicle windows, containers, tableware, or head lamps. This task is motivated by forensic needs in criminal investigation. The data have 9 inputs, 6 outputs, and 214 examples.

\subsubsection{Soybean}

The task is to recognize 19 different diseases of soybeans. The discrimination is done based on a description of bean (e.g. whether its size and color are normal), the plants (e.g. the size of spots on the leafs, whether these spots have a halo, whether plant growth is normal, whether roots are rotted), and information about the history of the plant's life (whether changes in crop occurred in the last year or last two years, whether seeds were treated, how the environment temperature is). There are 35 inputs, 19 outputs, and 683 examples in the data.

\subsection{Experimental setup}

All the datasets discussed above contain real-valued data. The inputs were phase encoded as discussed in section 2.1. Class labels were encoded by 1-of- $n$ encoding for $n$-class problem using output values 0 and 1 . To determine the class winner-takes-all method was used, i.e. for a given input pattern, the output neuron with the highest activation designated the class. Regarding the architecture of neural networks, the single-layered CVNN consisted of $n$ CVNs, while two-layer RVNN architecture was selected in such a way that the number of parameters for both the CVNN and RVNN were nearly equal. For CVNNs, each complex- 
valued weight was counted as two parameters since it has real and imaginary part. Table 6 shows the number parameters for CVNNs and RVNNs used in the experiments.

The backpropagation algorithm was used to train the RVNN, while the learning rules discussed in section 3 were used to train the CVNN. During the training, we kept the learning rate at 0.1 for both the networks. Weights and biases (in case of CVNN, real and imaginary parts) were initialized with random numbers from uniform distribution in the range of $[-0.5$, $0.5]$.

We used the partitioning of the dataset into training, validation, and test sets as given in Proben1 [14]. The partitioning is shown in Table 7. We trained both the networks over the training set for 1000 epochs, except for the Glass and Soybean problems. During these 1000 epochs, the weight parameters with the minimum mean squared error (MSE) over validation set, was stored and applied on test set. We calculated this validation error by using the same formulation as Eq. (16) below, except with the validation set instead of training set. Validation error was measured after every five epochs during the training process. For Glass problem we trained RVNN up to 5000 epochs, and for Soybean 4000 epochs. CVNN was trained up to 1000 epochs for all the datasets.

\subsection{Learning convergence}

When a neural network is trained with input patterns, the error on the training set decreases gradually with the epochs. We used the following formula to measure the mean squared training error over an epoch.

$$
E=\frac{1}{2 P} \sum_{p=1}^{P} \sum_{i=1}^{n}\left(d_{p i}-y_{p i}\right)^{2}
$$


where, $p$ is the pattern number, $P$ the total number of training patterns, and $n$ is the number of output neurons.

Fig. 6 shows how the mean squared training error changed over the epochs for two-layered RVNN, and single-layered CVNN with both the proposed activation functions on diabetes dataset. For both the activation functions, CVNN converged much faster then the RVNN. Especially, CVNN with the activation function 2 converged faster than with the activation function 1. In the following section we show it quantitatively for all the datasets used in the experiments.

\subsection{Results}

Here we compare the generalization ability of the single-layered CVNN and two-layered RVNN on the basis of misclassification on test dataset. We also compare the number of epochs during training to reach the minimum MSE over validation set as discussed in section 5.3. Table 8 summarizes the results. Results were taken over 20 independent runs for each of the datasets.

As shown in Table 8, the test set errors obtained with the activation function 1 tended to be more or less similar to those with the activation function 2. For six datasets, Cancer3, Diabetes2, Glass1, Glass2, Glass 3 and Soybean1, the average classification errors of CVNN were a little bit less than those of the RVNN, while for the others little a bit more than those

of the RVNN. Single-layered CVNNs, with both the activation functions, thus exhibit comparable generalization ability to that of RVNNs. 
Regarding the learning convergence, single-layered CVNNs required far less training cycles (epochs), in almost all the cases, to reach the minimum validation error than the RVNN counterpart as can be seen in Table 8 . In other words, learning convergence of CVNNs is faster than that of the RVNN. CVNNs with the activation function 2 seem to have even faster convergence than that of the activation function 1 in most of the cases.

In section 2.1 , we mentioned that both the interval $[0, \pi]$ and $[0, \pi / 2]$ may be used for the phase encoding of real-valued inputs. Table 9 shows the number epochs to reach the minimum validation error in both the encoding schemes, with each of the activation functions. As apparent from the table, the learning convergence is faster for the interval $[0, \pi]$ in most of the cases. The difference in generalization ability (test set error) was insignificant and thus the results are not shown.

\section{Discussion}

Two new activation functions for CVNs have been proposed in this study. These are suitable for applying single-layered CVNN to real-valued classification problems. It is shown that encoding real-valued inputs into phases, and processing information in complex domain, improves classification ability of a neuron. Capability of the proposed CVNs is illustrated by solving two-input Boolean functions, three-input Boolean functions, and symmetry detection in binary sequences of various lengths. A single-layered CVNN consisted of multiple CVNs with decision making in a winner-takes-all fashion is proposed and tested with real world benchmark problems. 
We explained graphically the nature of the proposed activation functions. Both the activation functions have four regions of saturation. We exploited three regions for activation function 1 , and all four regions for activation function 2 , since target outputs were given either 0 or 1 . Such saturation regions improve the classification ability of a CVN. Michel et al. [8] have reported that their model is able to solve 245 out of 256 three-input Boolean functions, while the proposed CVNs could solve 253 functions. Solving symmetry detection problem in binary sequences by a CVN up to six bits has been reported by Nitta [12]. We here showed that the proposed CVN can solve up to 10 bits. However, there is a limitation of output encoding for high dimensional symmetry detection problems. Using two CVNs, this problem can be solved to a considerable extent.

The single-layered CVNN is much faster in learning than the RVNN in terms of number of epochs for reaching minimum validation error. Generalization ability of the single-layered CVNN is comparable to two-layer RVNN on real world benchmark problems. One advantage of using such a single-layered CVNN instead of RVNN for classification problems is that the architecture selection problem does not exist in case of the single-layered CVNN.

Though there is no complex-valued activation function having differentiability and boundness at the same time in the entire complex domain, one can treat real and imaginary parts of net-input of a CVN individually, and then can combine them meaningfully. Also making dimensionality high (for example, quaternions) may be a new direction for enhancing the ability of neural networks. We think solving real-valued problems by CVNN more efficiently deserves further research. 


\section{References}

[1] I. Aizenberg, C. Moraga, Multilayer feedforward neural network based on multi-valued neurons and a backpropagation learning algorithm, Soft Computing, 11 (2) (2007) 169183.

[2] H. Aoki, Application of complex-valued neural networks for image processing, in: A. Hirose, ed., Complex-Valued Neural Networks: Theories and Applications, Vol. 5 (World Scientific Publishing, Singapore, 2003) 181-204.

[3] G. Georgiou, C. Koutsougeras, Complex backpropagation, IEEE Transactions on Circuits and Systems II, 39 (5) (1992) 330-334.

[4] A. Hirose, Applications of complex-valued neural networks to coherent optical computing using phase-sensitive detection scheme, Information Sciences - Applications, 2 (2) (1994) 103-117.

[5] A. Hirose, Complex-Valued Neural Networks (Springer-Verlag, Berlin, 2006).

[6] K. Hornik, M. Stinchombe, H. White, Multilayer Feedforward networks are universal approximators, Neural Networks, 2 (1989) 359-366.

[7] T. Kim, T. Adali, Fully complex multilayer perceptron for nonlinear signal processing, Journal of VLSI Signal Processing Systems for Signal, Image, and Video Technology, Special Issue: Neural Networks for Signal Processing, 32 (2002) 29-43.

[8] H. Leung, S. Haykin, The complex backpropagation algorithm, IEEE Transactions on Signal Processing, 39 (9) (1991) 2101-2104.

[9] H. E. Michel, A. A. S. Awwal, Enhanced artificial neural networks using complexnumbers, in: Proc. IJCNN'99 International Joint Conference on Neural Networks, Vol. 1 (1999) 456-461.

[10]T. Nitta, An extension of the backpropagation algorithm to complex numbers, Neural Networks, 10 (8) (1997) 1391-1415. 
[11]T. Nitta, On the inherent property of the decision boundary in complex-valued neural networks. Neurocomputing, 50 (C) (2003) 291-303.

[12]T. Nitta, Solving the XOR problem and the detection of symmetry using a single complex-valued neuron, Neural Networks, 16 (8) (2003) 1101-1105.

[13] T. Nitta, Three-dimensional vector valued neural network and its generalization ability. Neural Information Processing - Letters and Reviews, 10 (10) (2006) 237-242.

[14]L. Prechelt, PROBEN1 - A set of benchmarks and benchmarking rules for neural network training algorithms, University of Karlsruhe, Germany, Available FTP: (ftp.ira.uka.de/pub/neuron/proben1.tar.gz).

[15]A. B. Suksmono, A. Hirose, Adaptive interferometric radar image processing by complex-valued neural networks, in: A. Hirose, ed., Complex-Valued Neural Networks: Theories and Applications, Vol. 5 (World Scientific Publishing, Singapore, 2003) 277301.

[16]B. Widrow, J. McCool, M. Ball, The complex LMS algorithm, Proceedings of IEEE, 63 (4) (1975) 719-720.

[17] W. H. Wolberg, O. L. Mangasarian, Multisurface method of pattern separation for medical diagnosis applied to breast cytology, Proceedings of the National Academy of Sciences, 87 (1990) 9193-9196. 
Table 1

\begin{tabular}{|c|c|c|}
\hline \multicolumn{2}{|c|}{ Inputs } & \multirow{2}{*}{ Output } \\
\cline { 1 - 2 }$x_{1}$ & $x_{2}$ & $y$ \\
\hline $1+i 0$ & $1+i 0$ & 0 \\
\hline $1+i 0$ & $-1+i 0$ & 1 \\
\hline$-1+i 0$ & $1+i 0$ & 1 \\
\hline$-1+i 0$ & $-1+i 0$ & 0 \\
\hline
\end{tabular}


Table 2

\begin{tabular}{|c|c|c|c|c|c|c|}
\hline \multirow{2}{*}{$\begin{array}{l}\text { Function } \\
y_{1} y_{2} y_{3} y_{4}\end{array}$} & \multicolumn{3}{|c|}{ Activation 1} & \multicolumn{3}{|c|}{ Activation 2} \\
\hline & $\theta$ & $w_{1}$ & $w_{2}$ & $\theta$ & $w_{1}$ & $w_{2}$ \\
\hline 0000 & $-3.61-3.61 \mathrm{i}$ & $0.00+0.00 \mathrm{i}$ & $0.00+0.00 \mathrm{i}$ & $-0.15+0.33 \mathrm{i}$ & $-0.19-0.28 \mathrm{i}$ & $-0.33-0.34 \mathrm{i}$ \\
\hline 0001 & $-2.97-2.98 \mathrm{i}$ & $-1.83-1.99 \mathrm{i}$ & $-1.83-1.99 \mathrm{i}$ & $-2.53+0.17 i$ & $-0.96-2.68 \mathrm{i}$ & $2.26-1.13 \mathrm{i}$ \\
\hline 0010 & $-2.97-2.98 \mathrm{i}$ & $-1.79-2.03 \mathrm{i}$ & $1.79+2.03 \mathrm{i}$ & $1.58-2.03 \mathrm{i}$ & $-2.69-0.74 \mathrm{i}$ & $-0.29-2.64 \mathrm{i}$ \\
\hline 0011 & $-1.68-1.02 \mathrm{i}$ & $-1.93-2.59 \mathrm{i}$ & $0.00+0.00 \mathrm{i}$ & $-2.27+2.32 \mathrm{i}$ & $2.41-2.34 \mathrm{i}$ & $0.05+0.07 i$ \\
\hline 0100 & $-2.97-2.97 \mathrm{i}$ & $1.86+1.96 \mathrm{i}$ & $-1.86-1.96 \mathrm{i}$ & $-2.36+1.05 i$ & $1.03+2.74 i$ & $2.57-0.25 \mathrm{i}$ \\
\hline 0101 & $-1.25-1.47 \mathrm{i}$ & $0.03-0.03 i$ & $-2.36-2.14 \mathrm{i}$ & $-2.27+2.32 \mathrm{i}$ & $-0.03-0.03 i$ & $2.40-2.35 \mathrm{i}$ \\
\hline 0110 & $-2.83-2.81 \mathrm{i}$ & $-2.83+2.82 \mathrm{i}$ & $2.83-2.82 \mathrm{i}$ & $-0.08-0.06 \mathrm{i}$ & $1.72-2.24 \mathrm{i}$ & $-2.38+1.19 \mathrm{i}$ \\
\hline 0111 & $-1.64+0.39 \mathrm{i}$ & $-2.70-0.39 \mathrm{i}$ & $1.61-2.90 \mathrm{i}$ & $-1.74+1.98 \mathrm{i}$ & $-1.74+1.98 \mathrm{i}$ & $4.05-4.02 \mathrm{i}$ \\
\hline 1000 & $-2.95-2.96 \mathrm{i}$ & $2.46+1.37 \mathrm{i}$ & $2.46+1.37 \mathrm{i}$ & $-2.31+0.96 \mathrm{i}$ & $-2.48+0.33 i$ & $0.98+2.67 i$ \\
\hline 1001 & $-2.83-2.83 \mathrm{i}$ & $-2.83+2.83 \mathrm{i}$ & $-2.83+2.83 \mathrm{i}$ & $-0.06-0.07 i$ & $-2.13+2.64 \mathrm{i}$ & $-2.54+2.03 \mathrm{i}$ \\
\hline 1010 & $-1.66-1.05 \mathrm{i}$ & $-0.01+0.01 \mathrm{i}$ & $1.95+2.56 \mathrm{i}$ & $2.36-2.20 \mathrm{i}$ & $-0.08-0.08 \mathrm{i}$ & $2.31-2.46 \mathrm{i}$ \\
\hline 1011 & $1.58-1.89 \mathrm{i}$ & $-1.58-2.17 \mathrm{i}$ & $2.88-1.15 \mathrm{i}$ & $1.50-2.17 \mathrm{i}$ & $-4.10+3.98 i$ & $-1.50+2.17 \mathrm{i}$ \\
\hline 1100 & $-1.31-1.42 \mathrm{i}$ & $2.30+2.19 \mathrm{i}$ & $0.00+0.00 \mathrm{i}$ & $2.22-2.50 \mathrm{i}$ & $2.45-2.17 \mathrm{i}$ & $-0.07-0.03 i$ \\
\hline 1101 & $1.81-1.91 \mathrm{i}$ & $2.91-0.93 \mathrm{i}$ & $-1.81-1.95 \mathrm{i}$ & $-4.01+4.07 i$ & $-1.99+1.74 i$ & $1.99-1.74 \mathrm{i}$ \\
\hline 1110 & $-1.05-0.84 \mathrm{i}$ & $2.82-0.84 \mathrm{i}$ & $-1.05+2.83 i$ & $-2.25+1.38 \mathrm{i}$ & $2.25-1.38 \mathrm{i}$ & $-3.95+4.13 \mathrm{i}$ \\
\hline 1111 & $1.11+0.57 \mathrm{i}$ & $0.08-0.09 \mathrm{i}$ & $-0.19+0.21 \mathrm{i}$ & $-4.66+4.67 i$ & $0.02+0.01 \mathrm{i}$ & $-0.01+0.00 \mathrm{i}$ \\
\hline
\end{tabular}


Table 3

\begin{tabular}{|c|c|c|c|}
\hline \multicolumn{3}{|c|}{ Inputs } & Output \\
\hline$x_{1}$ & $x_{2}$ & $x_{3}$ & $\mathrm{y}$ \\
\hline 0 & 0 & 0 & 1 \\
\hline 0 & 0 & 1 & 0 \\
\hline 0 & 1 & 0 & 1 \\
\hline 0 & 1 & 1 & 0 \\
\hline 1 & 0 & 0 & 0 \\
\hline 1 & 0 & 1 & 1 \\
\hline 1 & 1 & 0 & 0 \\
\hline 1 & 1 & 1 & 1 \\
\hline
\end{tabular}


Table 4

\begin{tabular}{|c|c|c|c|}
\hline \multirow{2}{*}{ Length } & \multirow{2}{*}{ Sym. ${ }^{1}+$ Asym. ${ }^{2}$} & \multicolumn{2}{|c|}{ Misclassified. ${ }^{3} /$ Total patterns } \\
\hline & & Activation 1 & Activation 2 \\
\hline 2 & $2+2$ & $0 / 4$ & $0 / 4$ \\
\hline 3 & $4+4$ & $0 / 8$ & $0 / 8$ \\
\hline 4 & $4+12$ & $0 / 16$ & $0 / 16$ \\
\hline 5 & $8+24$ & $0 / 32$ & $0 / 32$ \\
\hline 6 & $8+56$ & $0 / 64$ & $0 / 64$ \\
\hline 7 & $16+112$ & $0 / 128$ & $0.8 / 128$ \\
\hline 8 & $16+240$ & $16 / 256$ & $6.6 / 256$ \\
\hline 9 & $32+480$ & $32 / 512$ & $8.8 / 512$ \\
\hline 10 & $32+992$ & $32 / 1024$ & $32 / 1024$ \\
\hline
\end{tabular}


Table 5

\begin{tabular}{|c|c|c|c|c|c|}
\hline $\begin{array}{c}\text { Bit } \\
\text { patterns }\end{array}$ & $\begin{array}{c}\text { Neuron } 1 \\
\text { Output(target) }\end{array}$ & $\begin{array}{c}\text { Neuron } 2 \\
\text { Output(target) }\end{array}$ & $\begin{array}{c}\text { Bit } \\
\text { patterns }\end{array}$ & $\begin{array}{c}\text { Neuron } 1 \\
\text { Output(target) }\end{array}$ & $\begin{array}{c}\text { Neuron } 2 \\
\text { Output(target) }\end{array}$ \\
\hline 0000000 & $0.942(1)$ & $0.548(0)$ & 1000000 & $0.000(0)$ & $0.528(1)$ \\
\hline 0000001 & $0.000(0)$ & $0.532(1)$ & 1000001 & $0.942(1)$ & $0.512(0)$ \\
\hline 0000010 & $0.000(0)$ & $0.947(1)$ & 1000010 & $0.000(0)$ & $0.943(1)$ \\
\hline 0000011 & $0.000(0)$ & $0.944(1)$ & 1000011 & $0.000(0)$ & $0.940(1)$ \\
\hline 0000100 & $0.033(0)$ & $0.948(1)$ & 1000100 & $0.000(0)$ & $0.944(1)$ \\
\hline 0000101 & $0.000(0)$ & $0.945(1)$ & 1000101 & $0.033(0)$ & $0.941(1)$ \\
\hline 0000110 & $0.038(0)$ & $0.993(1)$ & 1000110 & $0.000(0)$ & $0.992(1)$ \\
\hline 0000111 & $0.000(0)$ & $0.992(1)$ & 1000111 & $0.038(0)$ & $0.992(1)$ \\
\hline 0001000 & $0.942(1)$ & $0.546(0)$ & 1001000 & $0.000(0)$ & $0.526(1)$ \\
\hline 0001001 & $0.000(0)$ & $0.530(1)$ & 1001001 & $0.942(1)$ & $0.510(0)$ \\
\hline 0001010 & $0.000(0)$ & $0.947(1)$ & 1001010 & $0.000(0)$ & $0.943(1)$ \\
\hline 0001011 & $0.000(0)$ & $0.944(1)$ & 1001011 & $0.000(0)$ & $0.940(1)$ \\
\hline 0001100 & $0.033(0)$ & $0.948(1)$ & 1001100 & $0.000(0)$ & $0.944(1)$ \\
\hline 0001101 & $0.000(0)$ & $0.945(1)$ & 1001101 & $0.033(0)$ & $0.941(1)$ \\
\hline 0001110 & $0.038(0)$ & $0.993(1)$ & 1001110 & $0.000(0)$ & $0.992(1)$ \\
\hline 0001111 & $0.000(0)$ & $0.992(1)$ & 1001111 & $0.038(0)$ & $0.991(1)$ \\
\hline 0010000 & $0.033(0)$ & $0.948(1)$ & 1010000 & $0.000(0)$ & $0.944(1)$ \\
\hline 0010001 & $0.000(0)$ & $0.945(1)$ & 1010001 & $0.033(0)$ & $0.941(1)$ \\
\hline 0010010 & $0.000(0)$ & $0.993(1)$ & 1010010 & $0.000(0)$ & $0.992(1)$ \\
\hline 0010011 & $0.000(0)$ & $0.992(1)$ & 1010011 & $0.000(0)$ & $0.992(1)$ \\
\hline 0010100 & $0.942(1)$ & $0.993(0)$ & 1010100 & $0.000(0)$ & $0.992(1)$ \\
\hline 0010101 & $0.000(0)$ & $0.992(1)$ & 1010101 & $0.942(1)$ & $0.992(0)$ \\
\hline 0010110 & $0.000(0)$ & $0.953(1)$ & 1010110 & $0.000(0)$ & $0.949(1)$ \\
\hline 0010111 & $0.000(0)$ & $0.950(1)$ & 1010111 & $0.000(0)$ & $0.946(1)$ \\
\hline 0011000 & $0.033(0)$ & $0.948(1)$ & 1011000 & $0.000(0)$ & $0.944(1)$ \\
\hline 0011001 & $0.000(0)$ & $0.945(1)$ & 1011001 & $0.033(0)$ & $0.941(1)$ \\
\hline 0011010 & $0.000(0)$ & $0.993(1)$ & 1011010 & $0.000(0)$ & $0.992(1)$ \\
\hline 0011011 & $0.000(0)$ & $0.992(1)$ & 1011011 & $0.000(0)$ & $0.991(1)$ \\
\hline 0011100 & $0.942(1)$ & $0.993(0)$ & 1011100 & $0.000(0)$ & $0.992(1)$ \\
\hline 0011101 & $0.000(0)$ & $0.992(1)$ & 1011101 & $0.942(1)$ & $0.992(0)$ \\
\hline 0011110 & $0.000(0)$ & $0.952(1)$ & 1011110 & $0.000(0)$ & $0.949(1)$ \\
\hline 0011111 & $0.000(0)$ & $0.950(1)$ & 1011111 & $0.000(0)$ & $0.946(1)$ \\
\hline 0100000 & $0.000(0)$ & $0.947(1)$ & 1100000 & $0.000(0)$ & $0.943(1)$ \\
\hline 0100001 & $0.000(0)$ & $0.944(1)$ & 1100001 & $0.000(0)$ & $0.940(1)$ \\
\hline 0100010 & $0.942(1)$ & $0.992(0)$ & 1100010 & $0.000(0)$ & $0.992(1)$ \\
\hline 0100011 & $0.000(0)$ & $0.992(1)$ & 1100011 & $0.942(1)$ & $0.991(0)$ \\
\hline 0100100 & $0.000(0)$ & $0.993(1)$ & 1100100 & $0.000(0)$ & $0.992(1)$ \\
\hline 0100101 & $0.000(0)$ & $0.992(1)$ & 1100101 & $0.000(0)$ & $0.992(1)$ \\
\hline 0100110 & $0.033(0)$ & $0.953(1)$ & 1100110 & $0.000(0)$ & $0.950(1)$ \\
\hline 0100111 & $0.000(0)$ & $0.951(1)$ & 1100111 & $0.033(0)$ & $0.947(1)$ \\
\hline 0101000 & $0.000(0)$ & $0.946(1)$ & 1101000 & $0.000(0)$ & $0.943(1)$ \\
\hline 0101001 & $0.000(0)$ & $0.944(1)$ & 1101001 & $0.000(0)$ & $0.940(1)$ \\
\hline 0101010 & $0.942(1)$ & $0.992(0)$ & 1101010 & $0.000(0)$ & $0.992(1)$ \\
\hline 0101011 & $0.000(0)$ & $0.992(1)$ & 1101011 & $0.942(1)$ & $0.991(0)$ \\
\hline 0101100 & $0.000(0)$ & $0.992(1)$ & 1101100 & $0.000(0)$ & $0.992(1)$ \\
\hline 0101101 & $0.000(0)$ & $0.992(1)$ & 1101101 & $0.000(0)$ & $0.991(1)$ \\
\hline 0101110 & $0.033(0)$ & $0.953(1)$ & 1101110 & $0.000(0)$ & $0.949(1)$ \\
\hline 0101111 & $0.000(0)$ & $0.950(1)$ & 1101111 & $0.033(0)$ & $0.947(1)$ \\
\hline 0110000 & $0.038(0)$ & $0.993(1)$ & 1110000 & $0.000(0)$ & $0.992(1)$ \\
\hline 0110001 & $0.000(0)$ & $0.992(1)$ & 1110001 & $0.038(0)$ & $0.992(1)$ \\
\hline 0110010 & $0.033(0)$ & $0.953(1)$ & 1110010 & $0.000(0)$ & $0.950(1)$ \\
\hline 0110011 & $0.000(0)$ & $0.951(1)$ & 1110011 & $0.033(0)$ & $0.947(1)$ \\
\hline 0110100 & $0.000(0)$ & $0.953(1)$ & 1110100 & $0.000(0)$ & $0.949(1)$ \\
\hline 0110101 & $0.000(0)$ & $0.950(1)$ & 1110101 & $0.000(0)$ & $0.946(1)$ \\
\hline 0110110 & $0.942(1)$ & $0.541(0)$ & 1110110 & $0.000(0)$ & $0.520(1)$ \\
\hline 0110111 & $0.000(0)$ & $0.525(1)$ & 1110111 & $0.942(1)$ & $0.503(0)$ \\
\hline 0111000 & $0.038(0)$ & $0.992(1)$ & 1111000 & $0.000(0)$ & $0.992(1)$ \\
\hline 0111001 & $0.000(0)$ & $0.992(1)$ & 1111001 & $0.038(0)$ & $0.991(1)$ \\
\hline 0111010 & $0.033(0)$ & $0.953(1)$ & 1111010 & $0.000(0)$ & $0.949(1)$ \\
\hline 0111011 & $0.000(0)$ & $0.950(1)$ & 1111011 & $0.033(0)$ & $0.947(1)$ \\
\hline 0111100 & $0.000(0)$ & $0.952(1)$ & 1111100 & $0.000(0)$ & $0.949(1)$ \\
\hline 0111101 & $0.000(0)$ & $0.950(1)$ & 1111101 & $0.000(0)$ & $0.946(1)$ \\
\hline 0111110 & $0.942(1)$ & $0.539(0)$ & 1111110 & $0.000(0)$ & $0.517(1)$ \\
\hline 0111111 & $0.000(0)$ & $0.523(1)$ & 1111111 & $0.942(1)$ & $0.501(0)$ \\
\hline
\end{tabular}


Table 6

\begin{tabular}{|c|c|c|c|c|c|}
\hline \multicolumn{3}{|c|}{ Dataset } & CVNN & \multicolumn{2}{c|}{ RVNN } \\
\hline Name & Input & Output & Parameters & Hidden units & Parameters \\
\hline cancer & 9 & 2 & 40 & 3 & 38 \\
\hline card & 51 & 2 & 208 & 4 & 218 \\
\hline diabetes & 8 & 2 & 36 & 3 & 35 \\
\hline glass & 9 & 6 & 120 & 7 & 118 \\
\hline soybean & 82 & 19 & 3154 & 31 & 3181 \\
\hline
\end{tabular}


Table 7

\begin{tabular}{|c|c|c|c|c|}
\hline Dataset & $\begin{array}{c}\text { Total } \\
\text { patterns }\end{array}$ & $\begin{array}{c}\text { Training } \\
\text { set }\end{array}$ & $\begin{array}{c}\text { Validation } \\
\text { set }\end{array}$ & $\begin{array}{c}\text { Test } \\
\text { set }\end{array}$ \\
\hline cancer & 699 & 350 & 175 & 174 \\
\hline card & 690 & 345 & 173 & 172 \\
\hline diabetes & 768 & 384 & 192 & 192 \\
\hline glass & 214 & 107 & 54 & 53 \\
\hline soybean & 683 & 342 & 171 & 170 \\
\hline
\end{tabular}


Table 8

\begin{tabular}{|c|c|c|c|c|c|c|}
\hline \multirow{2}{*}{ Dataset } & \multicolumn{3}{|c|}{ Test set error \pm S.D. } & \multicolumn{3}{|c|}{ Epoch at min validation error } \\
\cline { 2 - 4 } & \multirow{2}{*}{ RVNN } & \multicolumn{2}{|c|}{ CVNN } & \multirow{2}{*}{ RVNN } & \multicolumn{2}{c|}{ CVNN } \\
\cline { 3 - 7 } & & Act. 1 & Act. 2 & & Act. 1 & Act. 2 \\
\hline cancer1 & $1.44 \pm 0.29$ & $2.56 \pm 0.29$ & $2.50 \pm 0.28$ & 133.25 & 30.00 & 23.25 \\
\hline cancer2 & $3.71 \pm 0.35$ & $4.37 \pm 0.29$ & $4.48 \pm 0.30$ & 69.00 & 21.25 & 16.25 \\
\hline cancer3 & $4.48 \pm 0.35$ & $4.02 \pm 0.00$ & $4.02 \pm 0.00$ & 69.25 & 27.25 & 20.25 \\
\hline card1 & $14.48 \pm 0.75$ & $14.94 \pm 0.80$ & $14.80 \pm 0.61$ & 30.75 & 7.25 & 6.00 \\
\hline card2 & $17.94 \pm 0.76$ & $17.82 \pm 1.07$ & $17.79 \pm 1.17$ & 16.25 & 4.50 & 6.25 \\
\hline card3 & $19.13 \pm 0.96$ & $18.98 \pm 1.20$ & $19.16 \pm 1.58$ & 33.75 & 10.25 & 10.00 \\
\hline diabetes 1 & $23.72 \pm 0.60$ & $25.60 \pm 0.39$ & $25.57 \pm 0.50$ & 630.25 & 83.75 & 40.00 \\
\hline diabetes2 & $26.17 \pm 0.84$ & $23.78 \pm 0.80$ & $24.71 \pm 0.85$ & 313.75 & 94.50 & 45.50 \\
\hline diabetes3 & $21.90 \pm 0.64$ & $21.28 \pm 0.76$ & $21.02 \pm 0.59$ & 416.25 & 7.00 & 6.00 \\
\hline glass1 & $35.28 \pm 5.38$ & $32.64 \pm 1.95$ & $29.72 \pm 3.67$ & 4590.50 & 194.00 & 131.25 \\
\hline glass2 & $53.87 \pm 1.67$ & $46.79 \pm 6.05$ & $44.72 \pm 5.61$ & 217.50 & 388.75 & 110.25 \\
\hline glass3 & $50.38 \pm 7.62$ & $39.06 \pm 5.71$ & $40.75 \pm 5.21$ & 4195.25 & 311.00 & 131.50 \\
\hline soybean1 & $10.62 \pm 1.23$ & $8.29 \pm 0.44$ & $9.32 \pm 1.62$ & 3545.50 & 220.75 & 209.75 \\
\hline soybean2 & $7.44 \pm 1.52$ & $6.88 \pm 0.57$ & $8.47 \pm 2.55$ & 3452.50 & 299.75 & 155.00 \\
\hline soybean3 & $8.65 \pm 1.54$ & $9.68 \pm 1.22$ & $10.18 \pm 2.50$ & 3122.25 & 140.00 & 116.25 \\
\hline
\end{tabular}


Table 9

\begin{tabular}{|c|c|c|c|c|}
\hline \multirow{2}{*}{ Data set } & \multicolumn{2}{|c|}{ Activation 1 } & \multicolumn{2}{c|}{ Activation 2 } \\
\cline { 2 - 5 } & $\pi / 2$ & $\pi$ & $\pi / 2$ & $\pi$ \\
\hline cancer1 & 61.25 & 30.00 & 42.00 & 23.25 \\
\hline cancer2 & 39.50 & 21.25 & 27.75 & 16.25 \\
\hline cancer3 & 50.75 & 27.25 & 38.25 & 20.25 \\
\hline card1 & 12.50 & 7.25 & 7.75 & 6.00 \\
\hline card2 & 6.00 & 4.50 & 6.00 & 6.25 \\
\hline card3 & 26.50 & 10.25 & 27.50 & 10.00 \\
\hline diabetes1 & 900.50 & 83.75 & 881.50 & 40.00 \\
\hline diabetes2 & 996.00 & 94.50 & 567.25 & 45.50 \\
\hline diabetes3 & 19.00 & 7.00 & 12.50 & 6.00 \\
\hline glass1 & 743.75 & 194.00 & 688.75 & 131.25 \\
\hline glass2 & 310.50 & 388.75 & 334.75 & 110.25 \\
\hline glass3 & 474.25 & 311.00 & 461.25 & 131.50 \\
\hline soybean1 & 376.00 & 220.75 & 400.50 & 209.75 \\
\hline soybean2 & 382.75 & 299.75 & 325.00 & 155.00 \\
\hline soybean3 & 215.00 & 140.00 & 259.50 & 116.25 \\
\hline
\end{tabular}




\section{Table Caption}

Table 1. Input-output mapping for XOR problem after phase encoding of inputs.

Table 2. Learned weights and bias of CVN for two-input Boolean functions.

Table 3. Input-output mapping of a symmetry detection problem with three inputs. Output 1 means corresponding input pattern is symmetric, and 0 means asymmetric.

Table 4. Average number of misclassifications by two CVNs for symmetry detection problems. Averages are taken over 10 independent runs. ${ }^{1},{ }^{2}$, and ${ }^{3}$ refer to the number of symmetric patterns, number of asymmetric patterns, and average number of misclassification, respectively.

Table 5. Examples of outputs of two CVNs with the activation function 2 for the seven-input symmetry detection problem in a single run.

Table 6. Number of parameters for single-layered CVNNs and two-layered RVNNs. Parameters include the connection weights and the biases of the neurons. For CVNN, each weight and bias are counted as two parameters, since they have real and imaginary parts.

Table 7. Total number of examples, and partitioning of the datasets into training, validation and test sets. 
Table 8. Average classification error on the test set, and the average number of epochs required to reach the minimum validation error. Averages are taken over 20 independent runs. Act. 1 and 2 refer to the activation function 1 and activation function 2, respectively.

Table 9. Average number of epochs required to reach the minimum validation error for the phase encoding $[0, \pi / 2]$ and $[0, \pi]$. Averages are taken over 20 independent runs. 


\section{Figure Captions}

Fig. 1. Model of a complex neuron. The $\operatorname{sign} \sum$ sums up the weighted inputs $w_{j} x_{j}(1 \leq j \leq m)$ and the bias $\theta . f_{C \rightarrow R}$ is an activation function that maps the complex-valued internal state to a real-valued output $y$.

Fig. 2. Phase encoded inputs. When a real value $x$ moves in the interval $[a, b]$, corresponding complex value moves over the upper half of unit circle. $x=a$, and $x=b$ are mapped by $e^{i 0}$, and $e^{i \pi}$ respectively.

Fig. 3. The proposed activation functions 1 and 2 that map complex-value $u+i v$ to real-value $y$. (a) Activation function 1 is the magnitude $\left|f_{R}(u)+i f_{R}(v)\right|$ of sigmoided real and imaginary parts, (b) Activation function 2 is the square of differences $\left(f_{R}(u)-f_{R}(v)\right)^{2}$ between sigmoided real and imaginary parts.

Fig. 4. Net-inputs of a CVN for XOR problem and contour diagram for the activation function 1 and 2 in (a) and (b), respectively. Curved lines indicate the points taking the same output values as indicated by the numbers. Horizontal and vertical lines are the real and imaginary axes, respectively. Cross mark $[\times]$ denotes the net-input for the input pattern belonging to class 1 (target output 1), and circle [O] denotes the net-input belonging to class 0 (target output 0 ). 
Fig. 5. Net-inputs of a single $\mathrm{CVN}$ for sixteen possible input patterns in the four-input symmetry detection problem on the contour diagrams of (a) the activation function 1, and (b) the activation function 2 .

Fig. 6. Learning processes of the two-layered RVNN, and single-layered CVNNs with the activation function 1 (CVNN Act1) and activation function 2 (CVNN Act2). 
Figure1

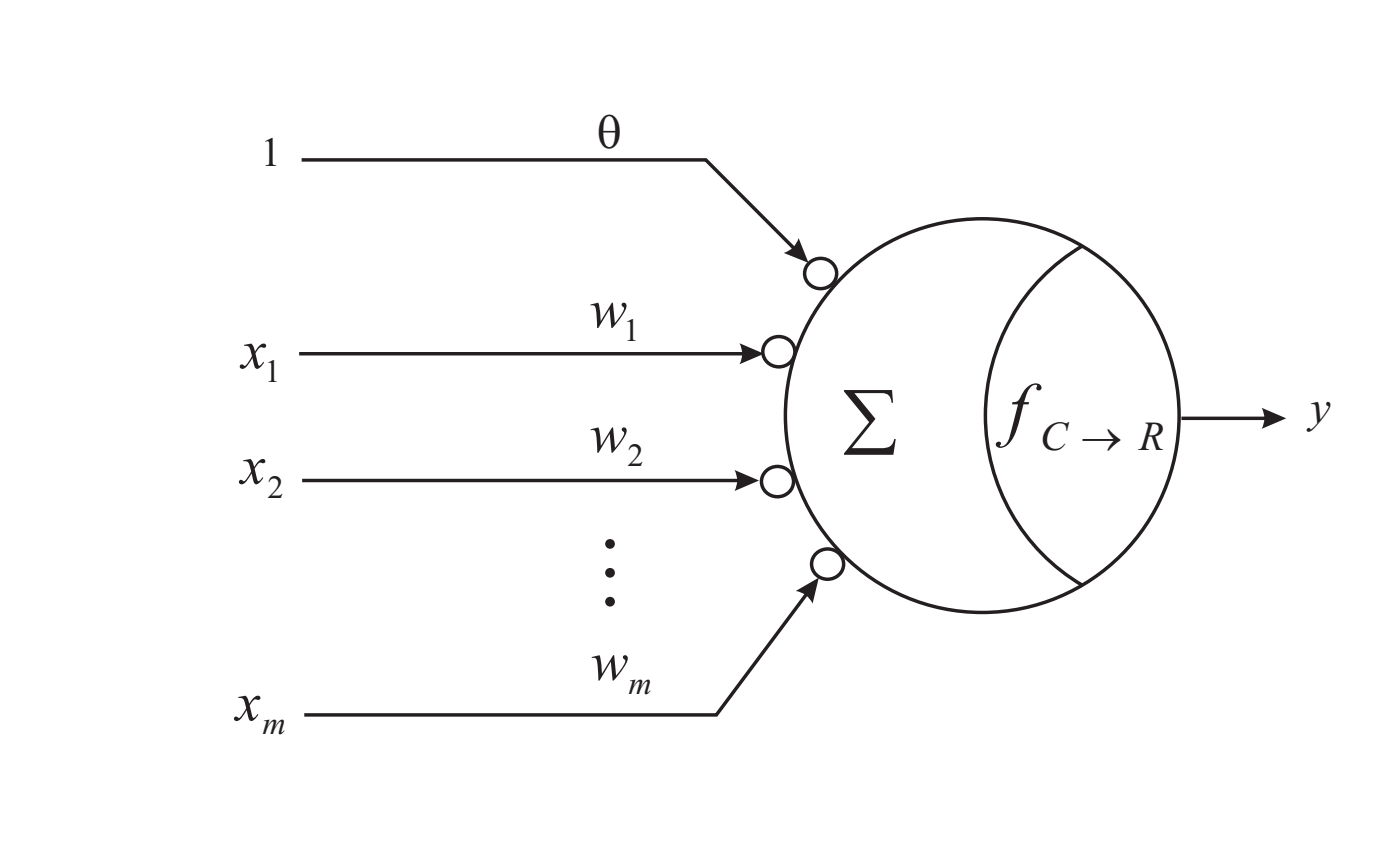

Figure
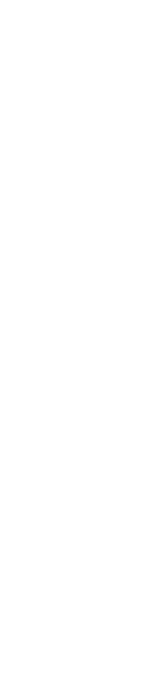

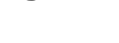
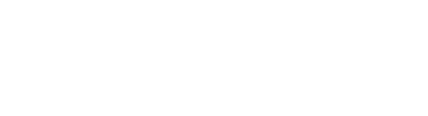
Figure2

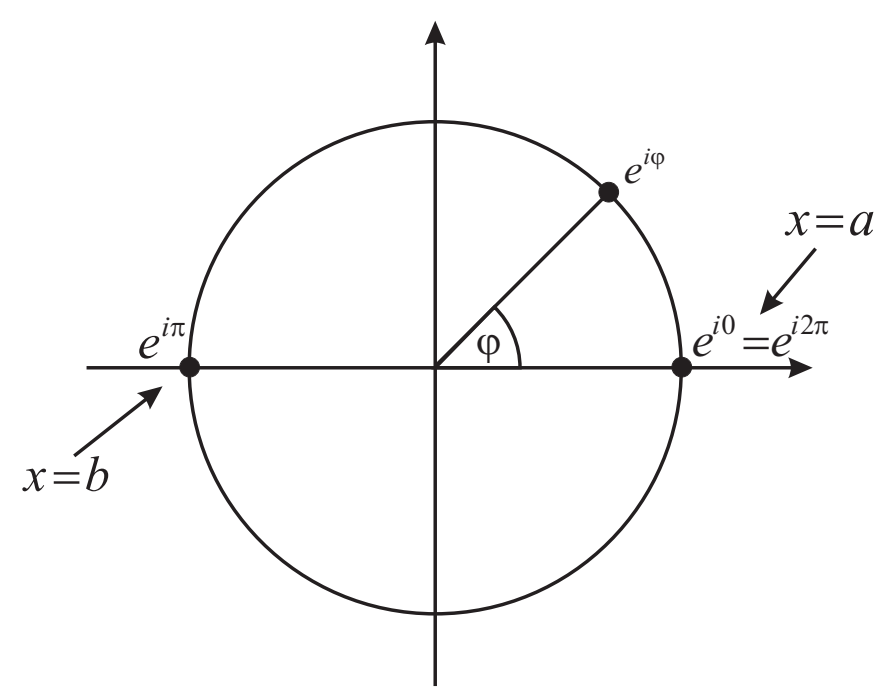


(a)

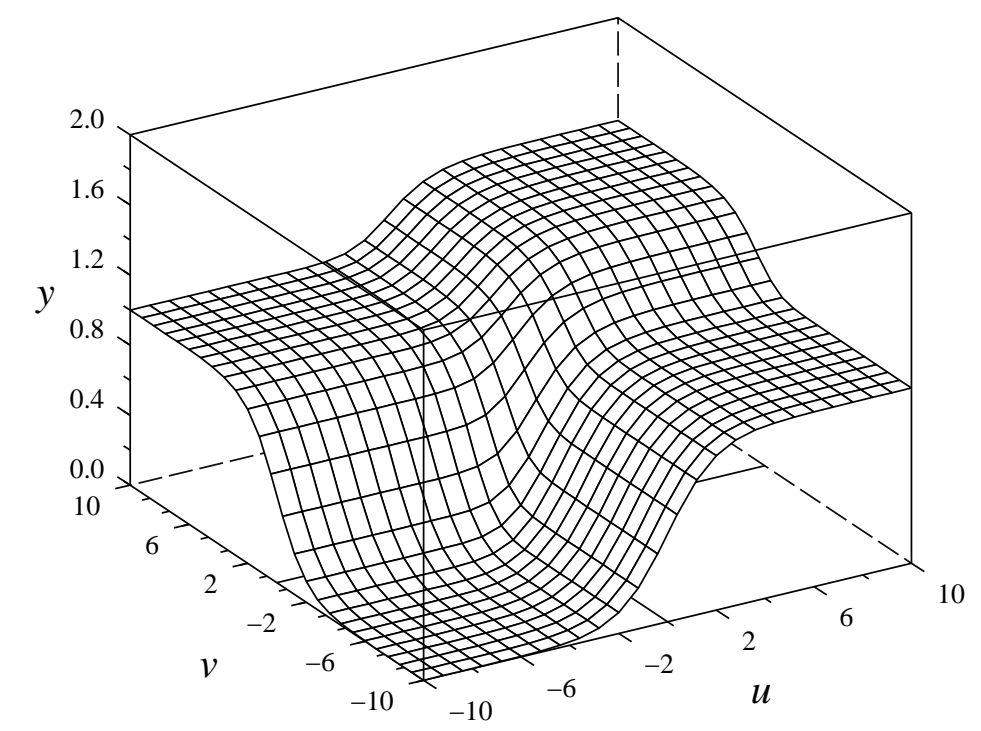

(b)

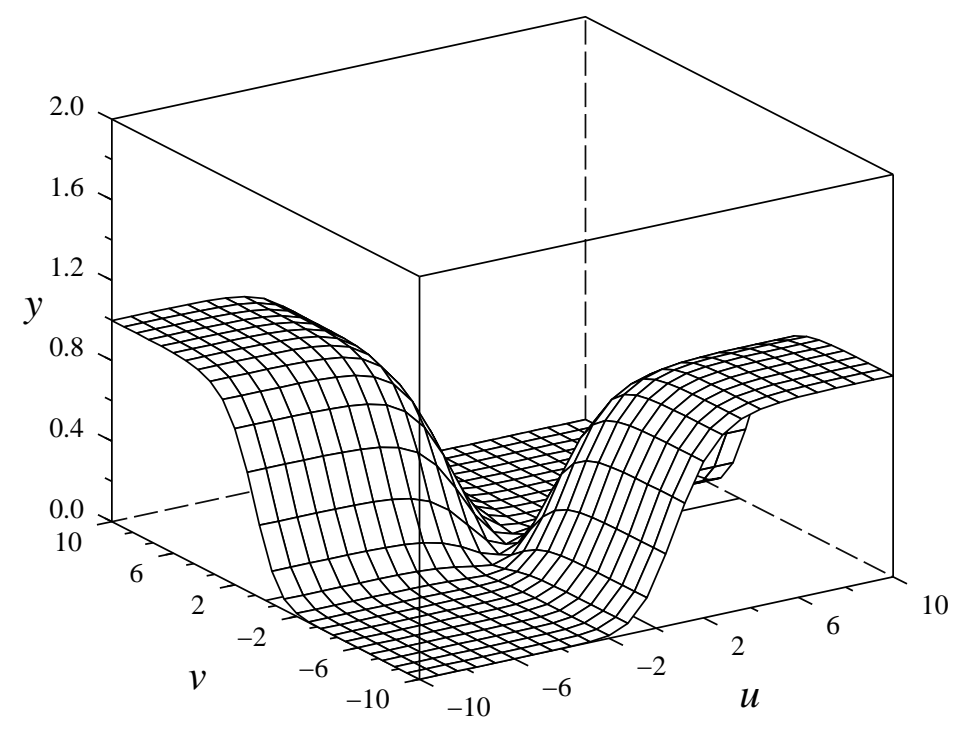


(b)

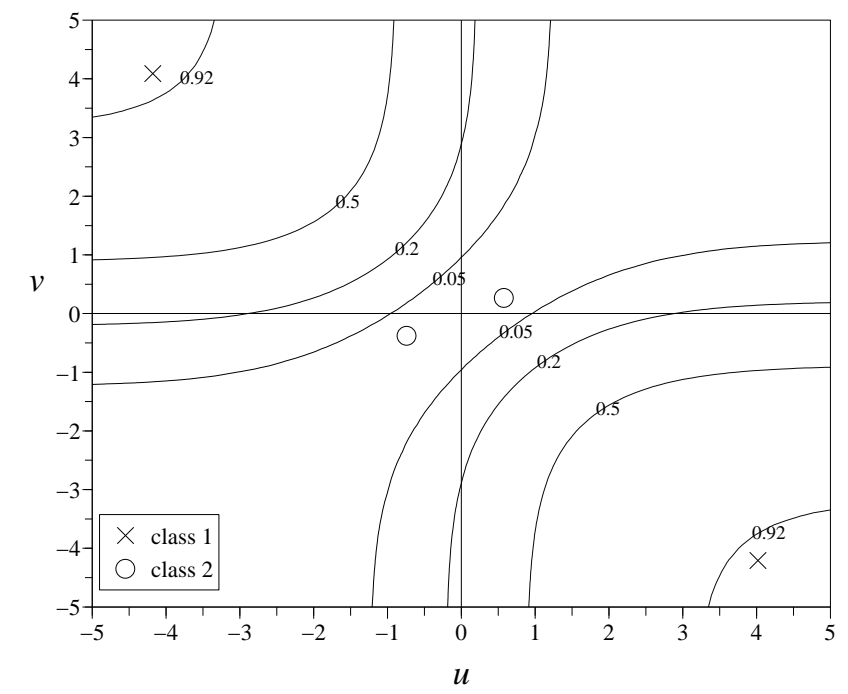

Figure4 (a)

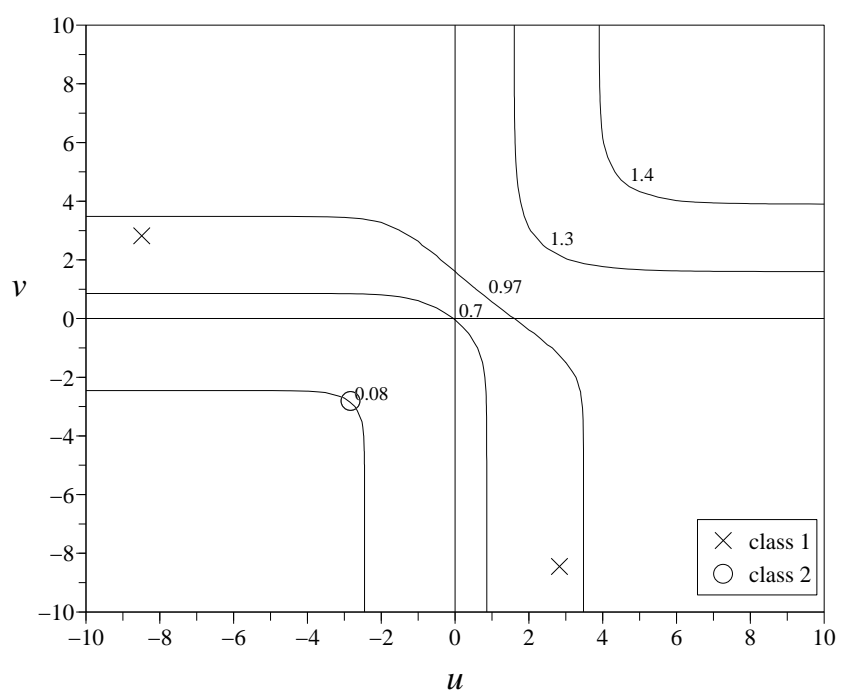

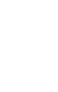


Figure5

(a)

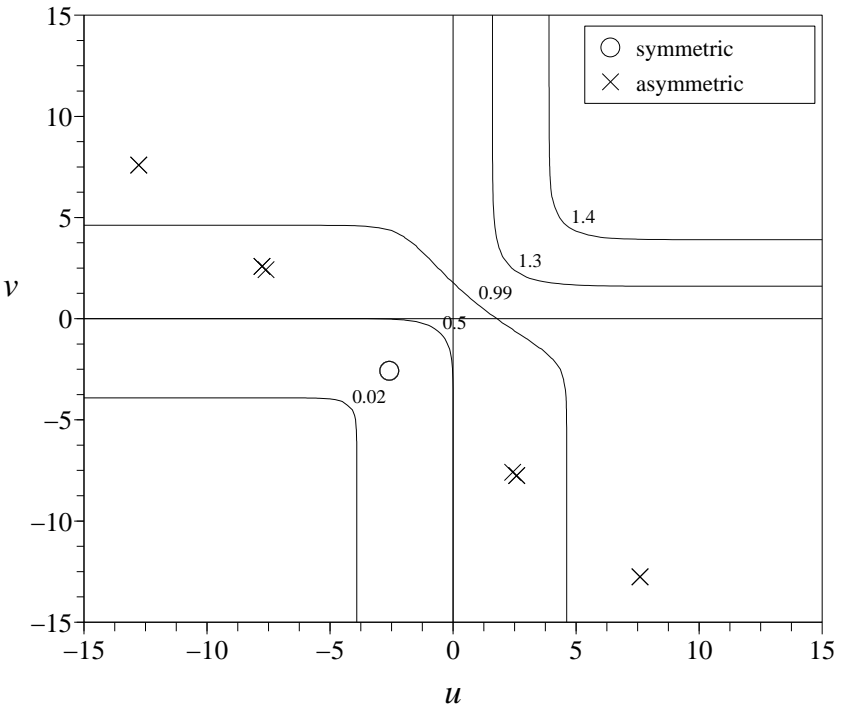

(b)

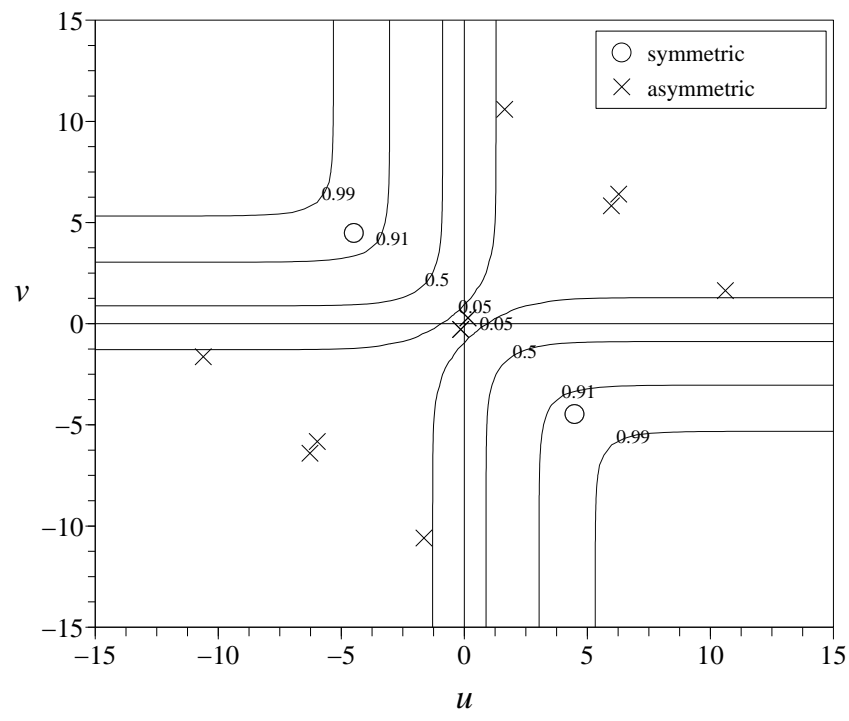




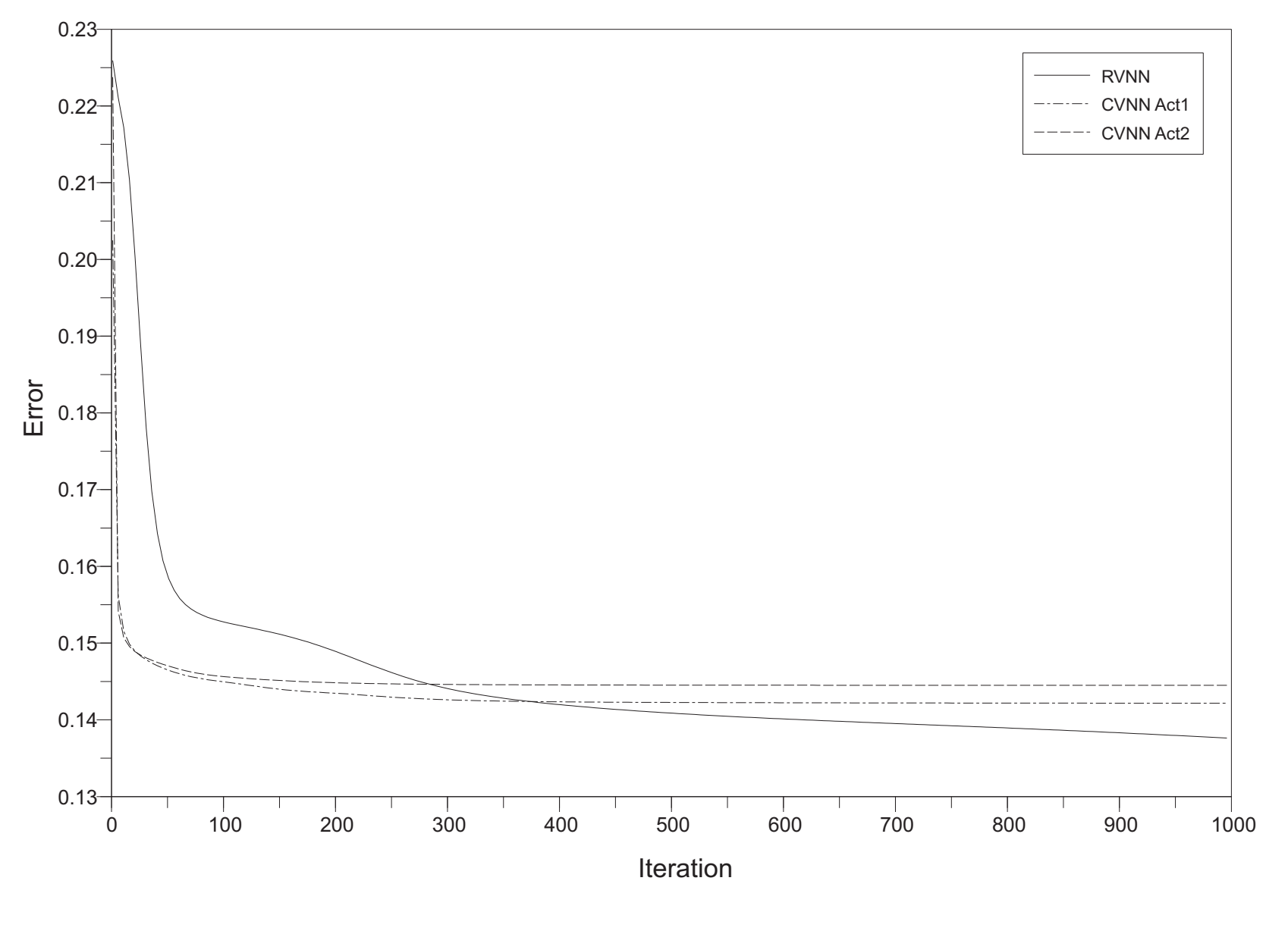

Figure6

.

\author{
reration
}

(n)

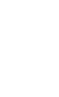
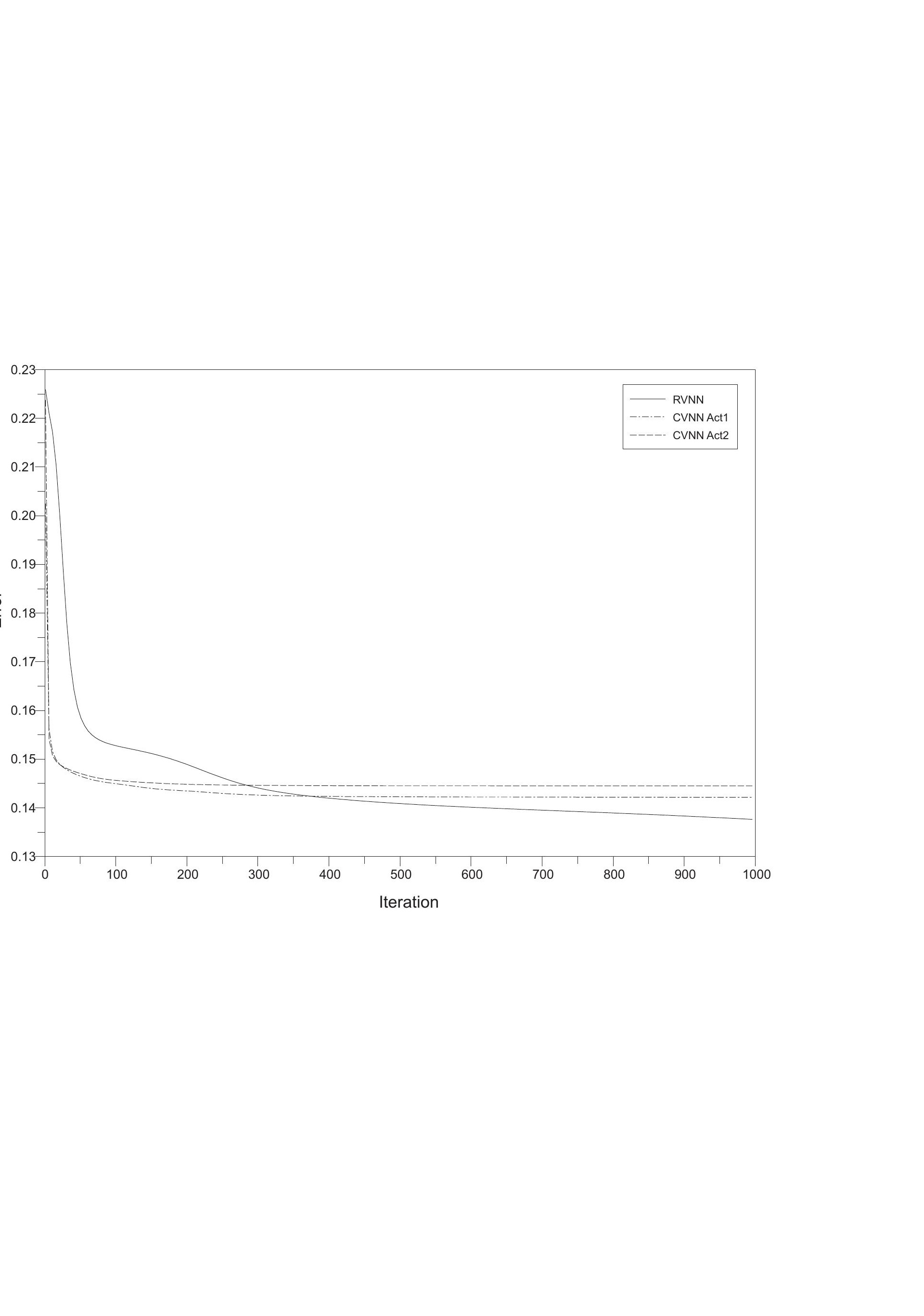